\title{
Ultrasound array photoacoustic microscopy for dynamic in vivo 3D imaging
}

Liang Song, Konstantin Maslov, K. Kirk Shung, Lihong V. Wang

Liang Song, Konstantin Maslov, K. Kirk Shung, Lihong V. Wang, "Ultrasound array photoacoustic microscopy for dynamic in vivo 3D imaging," Proc. SPIE 7564, Photons Plus Ultrasound: Imaging and Sensing 2010, 756403 (23 February 2010); doi: 10.1117/12.840582

SPIE. Event: SPIE BiOS, 2010, San Francisco, California, United States 


\title{
Ultrasound Array Photoacoustic Microscopy for Dynamic In Vivo 3-D Imaging
}

\author{
Liang Song ${ }^{1}$, Konstantin Maslov ${ }^{1}$, K. Kirk Shung ${ }^{2}$, and Lihong V. Wang ${ }^{1}$ \\ ${ }^{1}$ Optical Imaging Laboratory \\ Department of Biomedical Engineering \\ Washington University in St. Louis, St. Louis, Missouri 63130 \\ ${ }^{2}$ Department of Biomedical Engineering \\ University of Southern California, Los Angeles, California 90089
}

\begin{abstract}
Using realtime ultrasound array photoacoustic microscopy (UA-PAM), we demonstrated the feasibility of noninvasive in vivo imaging of human pulsatile dynamics, as well as 3-D dynamic imaging of sentinel lymph nodes (SLNs) in a murine model. The system, capable of realtime B-scan imaging at $50 \mathrm{~Hz}$ and high-speed 3-D imaging, was validated by imaging the subcutaneous microvasculature in rats and humans. After the validation, a human superficial palmar was imaged, and its pulsatile dynamics monitored, with 20-ms B-scan imaging temporal resolution. In addition, noninvasive photoacoustic sentinel lymph node (SLN) mapping with high spatial resolution has the potential to reduce the false negative rate and eliminate the use of radioactive tracers. Upon intra-dermal injection of Evans blue, the system maps SLNs accurately in mice and rats. Furthermore, the $\sim 6 \mathrm{~s} 3-\mathrm{D}$ imaging temporal resolution offers the capability to quantitatively and noninvasively monitor the dye dynamics in SLNs in vivo through sequential 3-D imaging. The demonstrated capability suggests that high-speed 3-D photoacoustic imaging should facilitate the understanding of the dynamics of various dyes in SLNs, and potentially help identify SLNs with high accuracy. With the results shown in this study, we believe that UA-PAM can potentially enable many new possibilities for studying functional and physiological dynamics in both preclinical and clinical imaging settings.
\end{abstract}

Keywords: photoacoustic microscopy, ultrasound array, dynamic in vivo imaging, three-dimensional imaging, microvascular imaging

\section{INTRODUCTION}

Owing to both the clinical needs and the demand for biomedical study of physiological dynamics, highspeed in vivo functional imaging has become highly desirable. Although MRI provides good functional imaging capability, it usually cannot perform real-time imaging [1]. Ultrasound offers real-time imaging capability, but the mechanical contrast does not provide much physiological information besides flow [2]. Previously available high-resolution optical microscopy modalities - including confocal microscopy [3], two-photon microscopy [4], and optical coherence tomography [5] - are capable of real-time imaging. However, as none of them sense optical absorption directly, their sensitivity for functional imaging is relatively low. Moreover, they rely on the detection of ballistic photons, and thus cannot image beyond one optical transport mean free path in highly scattering biological tissue.

Photoacoustic imaging is a noninvasive biomedical imaging technology that provides excellent optical absorption contrast - endogenous contrast for many physiological phenomena - with high ultrasonic resolution at super-depths - depths beyond the optical transport mean free path [6]. It has been used to

Photons Plus Ultrasound: Imaging and Sensing 2010, edited by Alexander A. Oraevsky, Lihong V. Wang, Proc. of SPIE Vol. 7564, 756403 · (C) 2010 SPIE · CCC code: 1605-7422/10/\$18 · doi: 10.1117/12.840582 
study whisker stimulation [7], single vessel oxygenation [8], and tumor hypoxia [9]. It is also a high-speed imaging modality by nature, with its speed fundamentally limited only by the photoacoustic wave propagation time. In principle, A-lines (i.e., depth-resolved 1-D images) can be acquired at $100 \mathrm{kHz}$ with a depth of $1.5 \mathrm{~cm}$.

Using a 30-MHz ultrasound array and a $\mathrm{kHz}$ repetition laser system, we developed photoacoustic microscopy capable of real-time B-scan imaging at $50 \mathrm{~Hz}$ and high-speed 3-D imaging, offering the feasibility to image physiological dynamics [10-12]. In addition, this ultrasound array photoacoustic microscopy (UA-PAM) system, with axial, lateral, and elevational resolutions of 25, 70, and $200 \mu \mathrm{m}$, respectively, provides $\sim 3 \mathrm{~mm}$ imaging depth in scattering biological tissue [10]. In this study, by imaging human pulsatile dynamics and murine sentinel lymph node dynamics, we demonstrated the system's dynamic functional imaging capability.

\section{IMAGING SYSTEM}

A schematic of the system is shown in Fig. 1. The key components include a diode-pumped Q-switched $\mathrm{Nd}$ :YLF laser, a tunable dye laser, a 30-MHz ultrasound array, an 8-channel PCI data acquisition (DAQ) card, a multi-core PC, and a custom designed light delivery system. Details of the system can be found in our previous publications $[10,11,13]$.

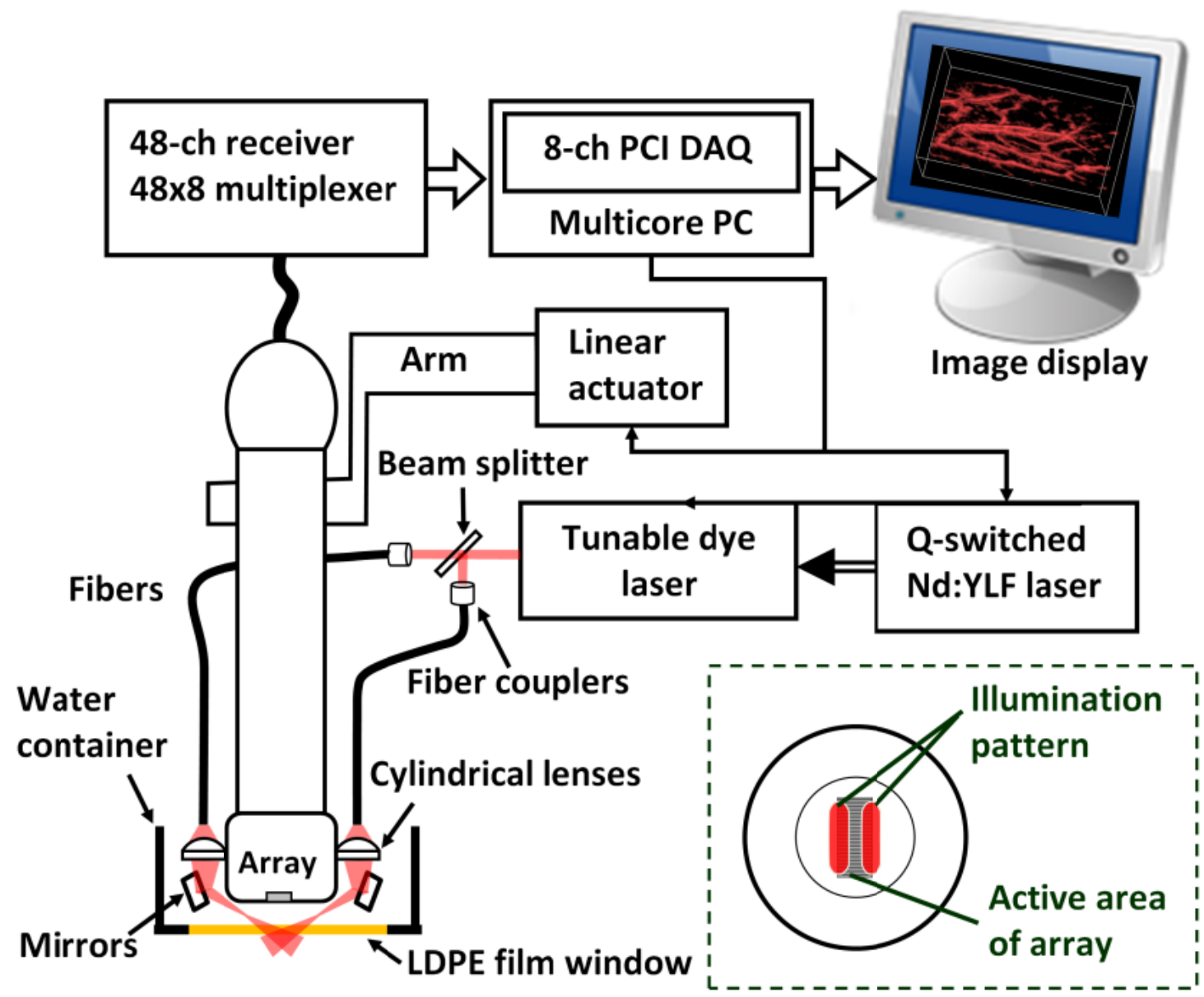

Fig. 1. Schematic of the ultrasound array photoacoustic microscopy system. Inset A, the dark-field illumination pattern on the skin surface. 


\section{RESULTS}

To validate the system's performance of in vivo microvascular imaging in both small animals and humans, we imaged the upper dorsal region of a Sprague Dawley rat (Harlan Sprague Dawley, Inc., USA), as well as a human palm. All the imaging procedures were conducted in compliance with the experimental protocols approved by Washington University in St. Louis.

Fig. 2 shows that blood vessels down to $\sim 100 \mu \mathrm{m}$ size were clearly imaged by the system. The acquisition time for each 3-D photoacoustic image was only $2 \mathrm{~s}$. Limited by the data transfer speed, the temporal resolution of sequential 3-D imaging was $\sim 6 \mathrm{~s}$.
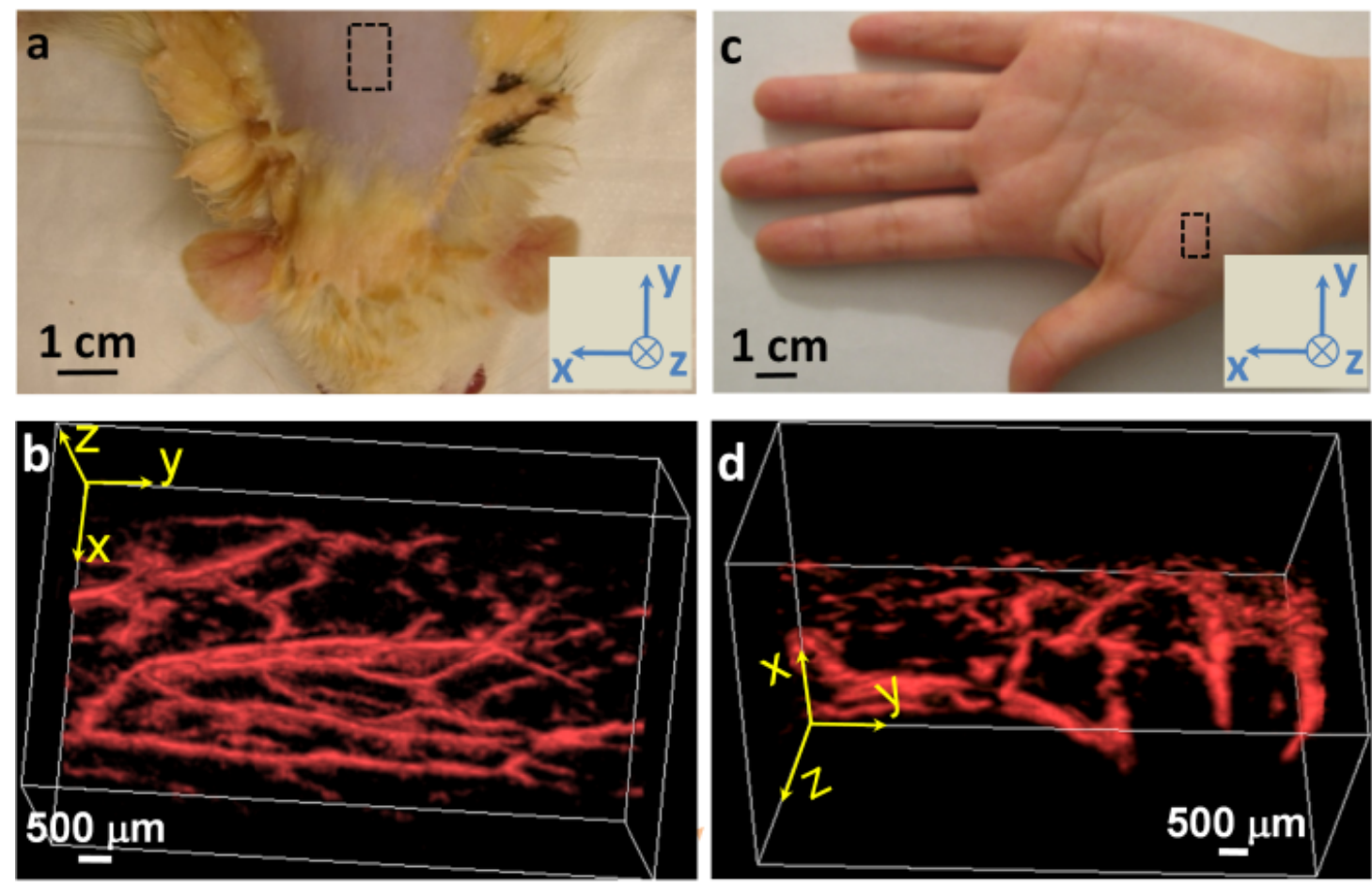

Fig. 2. (a) Photograph of the Sprague Dawley rat with hair removed before photoacoustic imaging. (b) In vivo photoacoustic 3-D image corresponding to the dashed rectangle area in (a). (c) Photograph of the imaged human palm. (d) In vivo photoacoustic 3-D image corresponding to the dashed rectangle area in (c).

In order to study the pulsatile dynamics, we scanned a region of the palm near the wrist, where the superficial palmar, $\sim 1 \mathrm{~mm}$ in diameter and over $1 \mathrm{~mm}$ deep, was imaged (Fig. 3). After acquiring the initial 3-D image, we fixed the scanning probe and continuously monitored the pulsation using B-scan (Video 1). The pulsatile rate, estimated from the image, was 66 per minute, consistent with the $65 \pm 2$ per-minute rate measured from a pulse oximeter. 

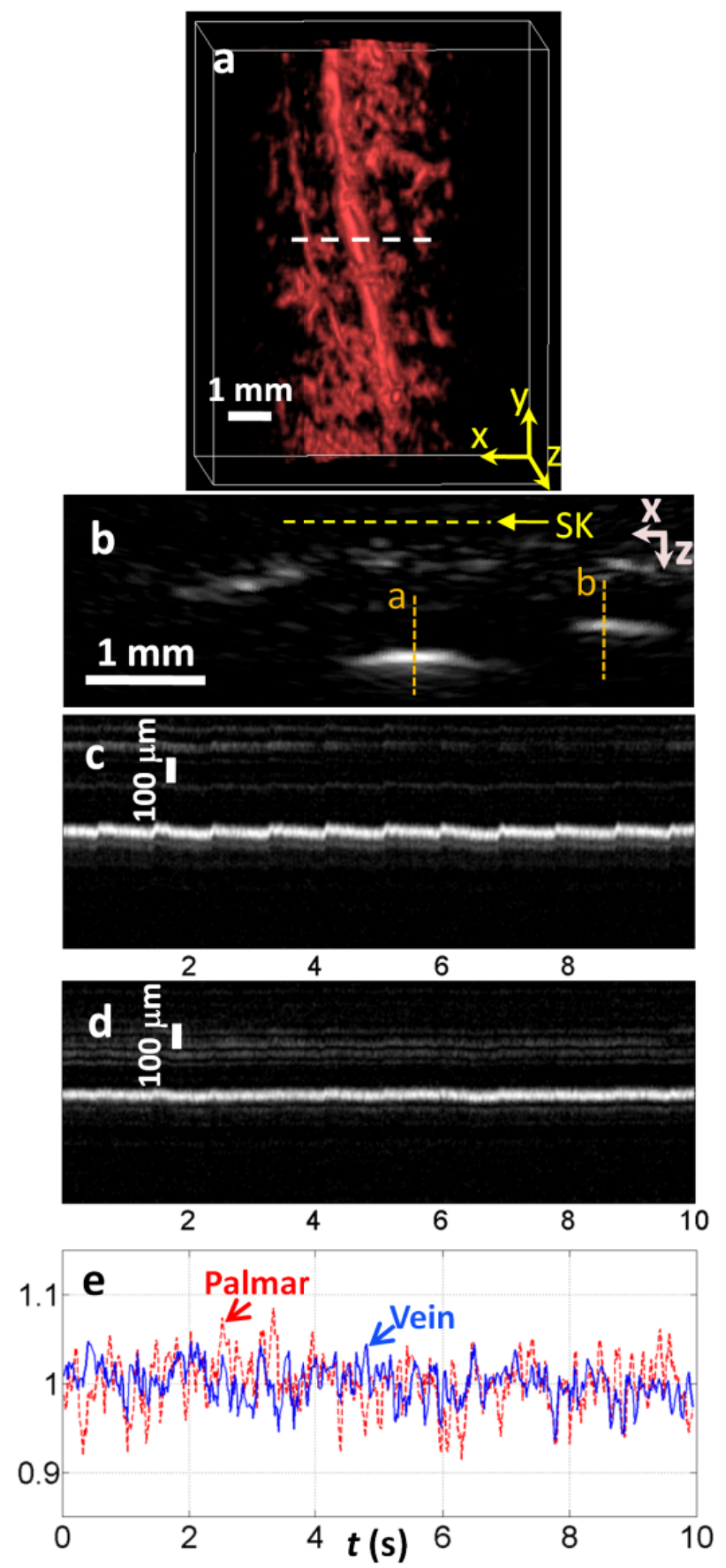

Fig. 3. In vivo photoacoustic imaging of human pulsatile dynamics. (a) 3-D photoacoustic image of the superficial palmar. The dashed line indicates the cross-section monitored by real-time B-scan imaging. (b) One B-scan slice from the real-time photoacoustic B-scan movie (http://dx.doi.org/10.1117/12.840582.1). SK, skin surface.

(c) M-mode image corresponding to the dotted vertical line $a$ in (b), showing the superficial palmar's pulsatile motion (A-line) as a function of time.

(d) M-mode image of a vein corresponding to the dotted vertical line $b$ in (b).

(e) Normalized integrated signal over the superficial palmar (red) and the vein (blue) regions as a function of time. 
Using a murine model, we also demonstrated the system's capability to accurately identify SLNs and quantitatively image the dye uptake and clearance dynamics [13].

\section{CONCLUSIONS AND DISCUSSION}

Our UA-PAM system was capable of high-speed in vivo imaging of microvasculature details in both small animals and humans. Using UA-PAM, human pulsatile dynamics was captured in real time. Ideally, to better characterize the pulsatile hemodynamics, oxygen saturation should be measured within each cardiac cycle. However, accurate computation of the blood oxygenation in real-time requires photoacoustic imaging with laser-wavelength tunability in real time (because oxygenation quantification requires multiwavelength measurements), which is currently not available in our laser system.

In addition, UA-PAM demonstrated accurate in vivo SLN mapping in a murine model. The dynamics of dye accumulation and clearance in murine SLNs were quantitatively monitored with a high temporal resolution, up to $6 \mathrm{~s}$. This capability should facilitate further studies to understand the dynamics of different dyes in SLNs, and potentially help identify SLNs with high accuracy.

With the promising results shown in this study, we believe that UA-PAM will open up many new possibilities for studying functional and physiological dynamics in both preclinical and clinical imaging settings.

\section{ACKNOWLEDGEMENTS}

This work was sponsored by grants R01 EB000712, R01 EB008085, R01 CA113453901, and U54 CA136398 (Network for Translational Research) from the National Institutes of Health. L.W. has a financial interest in Microphotoacoustics, Inc. and Endra, Inc., which, however, did not support this work.

\section{REFERENCES}

1. R. M. Heidemann, O. Ozsarlak, P. M. Parizel, J. Michiels, B. Kiefer, V. Jellus, M. Muller, F. Breuer, M. Blaimer, M. A. Griswold and P. M. Jakob, "A brief review of parallel magnetic resonance imaging," Eur Radiol 13(10), 2323-2337 (2003)

2. P. N. T. Wells, "Ultrasound imaging," Phys Med Biol 51(13), R83-R98 (2006)

3. R. H. Webb, "Confocal optical microscopy," Rep Prog Phys 59(3), 427-471 (1996)

4. W. Denk, J. H. Strickler and W. W. Webb, "Two-photon laser scanning fluorescence microscopy," Science 248(4951), 73-76 (1990)

5. D. Huang, E. A. Swanson, C. P. Lin, J. S. Schuman, W. G. Stinson, W. Chang, M. R. Hee, T. Flotte, K. Gregory, C. A. Puliafito and J. G. Fujimoto, "Optical Coherence Tomography," Science 254(5035), 1178$1181(1991)$

6. M. H. Xu and L. H. V. Wang, "Photoacoustic imaging in biomedicine," Review of Scientific Instruments 77(4), 041101 (2006)

7. X. D. Wang, Y. J. Pang, G. Ku, X. Y. Xie, G. Stoica and L. H. V. Wang, "Noninvasive laser-induced photoacoustic tomography for structural and functional in vivo imaging of the brain," Nat Biotechnol 21(7), 803-806 (2003)

8. H. F. Zhang, K. Maslov, G. Stoica and L. H. V. Wang, "Functional photoacoustic microscopy for highresolution and noninvasive in vivo imaging," Nat Biotechnol 24(7), 848-851 (2006)

9. M. L. Li, J. T. Oh, X. Y. Xie, G. Ku, W. Wang, C. Li, G. Lungu, G. Stoica and L. V. Wang, "Simultaneous molecular and hypoxia imaging of brain tumors in vivo using spectroscopic photoacoustic tomography," Proceedings of the Ieee 96(3), 481-489 (2008) 
10. L. Song, K. Maslov, R. Bitton, K. K. Shung and L. V. Wang, "Fast 3-D dark-field reflection-mode photoacoustic microscopy in vivo with a 30-MHz ultrasound linear array," J Biomed Opt 13(5), 054028 (2008)

11. R. J. Zemp, L. Song, R. Bitton, K. K. Shung and L. V. Wang, "Realtime photoacoustic microscopy in vivo with a 30-MHz ultrasound array transducer," Opt Express 16(11), 7915-7928 (2008)

12. R. J. Zemp, L. Song, R. Bitton, K. K. Shung and L. V. Wang, "Realtime photoacoustic microscopy of murine cardiovascular dynamics," Opt Express 16(22), 18551-18556 (2008)

13. L. Song, C. Kim, K. Maslov, K. K. Shung and L. V. Wang, "High-speed dynamic 3D photoacoustic imaging of sentinel lymph node in a murine model using an ultrasound array," Medical Physics 36(8), 3724-3729 (2009) 